\title{
„Die Radiologie nimmt eine Schlüsselposition für eine hochwertige Versorgung herzkranker Patientinnen und Patienten ein"
}

Die Umfrage „Status der kardiovaskulären Bildgebung in Deutschland“ untersucht, wie gut herzkranke Patientinnen und Patienten bundesweit mit kardiovaskulären radiologischen Leistungen versorgt sind. Über die Ergebnisse der Umfrage sowie die gesundheitspolitischen Forderungen, die sich aus ihr ergeben, haben wir mit dem Erstautor gesprochen, Dr. Malte Sieren, Radiologe am Universitätsklinikum Schleswig-Holstein, sowie seinen Mitautoren Univ.-Prof. Dr. David Maintz, Direktor des Instituts für Diagnostische und Interventionelle Radiologie an der Uniklinik Köln und Prof. Dr. Claas Philip Nähle, Oberarzt am Direktor Institut für Diagnostische und Interventionelle Radiologie an der Uniklinik Köln. Die Autoren sind in der Deutschen Röntgengesellschaft und dort unter anderem in der Arbeitsgemeinschaft „Herz- und Gefäßdiagnostik“ engagiert. Professor David Maintz steht der Arbeitsgemeinschaft als Vorsitzender vor.

Professor Nähle, unter welchen Herzerkrankungen leiden die Bundesbürgerinnen und Bundesbürger am häufigsten?

Die mit Abstand häufigste Erkrankung des Herz-Kreislauf-Systems ist der arterielle Blut- hochdruck, der dann - oft unbemerkt - zu Schäden an anderen Organen führt. Folgen können dann zum Beispiel Herzinfarkte durch Veränderungen (Engstellen) der Herzkranzgefäße und eine Herzleistungsschwäche (Herzinsuffizienz) sein. Aber auch andere Organe wie das Gehirn (Schlaganfälle) und die Nieren (Funktionseinschränkung) können betroffen sein. In den letzten Jahren sind darüber hinaus auch Erkrankungen der Herzklappen und Herzrhythmusstörungen häufiger geworden, nicht zuletzt auch durch die sich verändernde Altersstruktur der Bevölkerung. Insbesondere im ambulanten Bereich spielt auch die Herzmuskelentzündung, die Myokarditis, eine wichtige Rolle.

Professor Nähle, welche radiologischen Methoden werden bei der Versorgung von Herzerkrankten eingesetzt?

Heutzutage werden in der Radiologie die Herz-MRT und die Herz-CT eingesetzt. Welches Verfahren gewählt wird, hängt von der vermuteten Erkrankung beziehungsweise Fragestellung einerseits und dem Patienten andererseits ab. Hier die optimale Untersuchungsmethode und -technik zu wählen und anschließend zu interpretieren, ist die große Stärke der Radiologie. Hierbei ist die Bandbreite entsprechend der Vielzahl an Er- krankungen riesig und eine Aufzählung kann hier nur unvollständig bleiben. Während die MRT bei der Diagnostik der Herzmuskelentzündung mittlerweile - nicht zuletzt durch radiologische Studien - die Untersuchungsmethode der Wahl ist, rückt derzeit die Herz-CT zur Abklärung des sogenannten chronischen Koronarsyndroms einerseits, aber auch zur Therapiesteuerung mit den sogenannten Lipidsenkern bei bestimmten Patientengruppen andererseits in den Fokus. Weitere Untersuchungen, die wir regelmäßig durchführen, sind Kontrolluntersuchungen bei Patienten mit angeborenen Herzfehlern und Planungsuntersuchungen vor Herzklappeneingriffen.

Dr. Sieren, Sie haben mit Kollegen eine Umfrage und Analyse zum Thema „Status der kardiovaskulären Bildgebung in Deutschland“ durchgeführt. In Ihrem Paper zur Umfrage stellen Sie die Ergebnisse vor. Wie sind Sie bei der Umfrage vorgegangen und wer hat sich beteiligt?

Die Umfrage entstand vor dem Hintergrund der wachsenden Bedeutung der Herzbildgebung - die gerade durch die neuen ESC-Leitlinien zum Koronarsyndrom noch beeindruckend bestätigt wurde. Wir wollten wissen: Wie ist die Radiologie in 
Deutschland zur kardiovaskulären Bildgebung aufgestellt und sind wir den kommenden Herausforderungen gewachsen? Hierfür haben wir die umfassende Datenbank der DRG ausgewertet, haben einen Blick ins ESCR-Registry geworfen sowie eine Umfrage in der AG erarbeitet. Diese haben wir dann sowohl über Kanäle der DRG als auch des Berufsverbandes Deutscher Radiologen zirkuliert, um ein möglichst umfassendes Bild zu bekommen, vom Universitätsklinikum bis zur Niederlassung.

\section{Dr. Sieren, was sind die wichtigsten Er- gebnisse der Umfrage?}

Das zentrale Ergebnis der Umfrage ist, dass ein dichtes Netz aus radiologischen spezialisierten Zentren und Expertinnen und Experten in ganz Deutschland vorhanden ist, um die unmittelbare Versorgung mit hochqualitativer kardiovaskulärer Bildgebung sicherzustellen. So haben Patientinnen und Patienten nahezu überall unmittelbaren Zugang zu entsprechender Expertise und auch die Zuweiserinnen und Zuweiser haben lokale radiologische Ansprechpartnerinnen und Ansprechpartner. Bemerkenswert ist vor allem, dass alle Institutionstypen, von der Universitätsklinik bis zur Niederlassung, die Expertise bereitstellen und an der Versorgung teilnehmen. Die Befundung wird dabei sowohl von Radiologinnen und Radiologen als auch in enger interdisziplinärer Zusammenarbeit mit den klinischen Kolleginnen und Kollegen durchgeführt. Gerade bei einer technologisch hochanspruchsvollen Untersuchung wie der kardiologischen Schnittbildgebung nimmt die Radiologie als Brückendisziplin mit technischem und medizinischem Fachwissen sicherlich eine Schlüs- selposition ein, um die bestmögliche Versorgung von Patientinnen und Patienten zu gewährleisten.

Dr. Sieren, was tut die AG Herzdiagnostik der Deutschen Röntgengesellschaft zur Förderung der Herzbildgebung in der Radiologie?

Die gute Infrastruktur zur kardiovaskulären Bildgebung in Deutschland ist sicher auch ein wesentlicher Verdienst der Bemühungen der AG, das Fachwissen zur kardiovaskulären Bildgebung für Radiologinnen und Radiologen verfügbar zu machen. Von Kongressen - wie dem Röntgenkongress oder den interdisziplinären Kardiodiagnostiktagen - über die conrad-Fallsammlungen bis hin zu den Q-Kursen. Dabei wird versucht, das ganze Spektrum abzubilden, auch in Kooperation mit AGs außerhalb der kardiovaskulären Bildgebung. Einerseits bietet das gerade in Kooperation mit dem Forum Junge Radiologie fertiggestellte Weiterbildungscurriculum einen Einstieg in die kardiovaskuläre Bildgebung für junge Assistenzärztinnen und -ärzte. Andererseits wird auf Kongressen neuesten technologischen Entwicklungen eine Plattform geboten und zum Beispiel in Zusammenarbeit mit der AGIT die strukturierte Befundung weiterentwickelt. All diese Bemühungen fließen im Zertifizierungsprogramm der AG zusammen, und der Erfolg des Programms ist eine schöne Bestätigung dieser Initiativen.

Professor Maintz, die aktuellen Leitlinien zur Diagnostik und Therapie von Herzerkrankungen sehen teils einen häufigeren Einsatz der Schnittbildgebung vor als die Gesetzliche Krankenversicherung finan- ziert. Woran liegt das und wie könnte hier eine Lösung aussehen?

Wie Herr Professor Nähle bereits ausgeführt hat, hat sich die Herz-MRT mittlerweile zur Methode der Wahl bei der Diagnostik der Herzmuskelentzündung entwickelt, während die Herz-CT zur Abklärung der koronaren Herzkrankheit die erste Wahl ist. Dies spiegelt sich auch in einschlägigen Leitlinien wider, darunter zum Beispiel die Leitlinie der Europäischen Gesellschaft für Kardiologie zum chronischen Koronarsyndrom aus dem Jahr 2019. Für die Patientinnen und Patienten bedeutet das, dass eine leitliniengerechte Diagnostik häufig nichtinvasiv mit Methoden der Schnittbildgebung erfolgen soll statt mit deutlich belastenderen und komplikationsträchtigeren Katheteruntersuchungen. Trotz dieser Vorteile können Herz-CT und Herz-MRT aber aufgrund fehlender Vergütungsmöglichkeiten in der Gesetzlichen Krankenversicherung nicht allen Patientinnen und Patienten zugänglich gemacht werden, die laut Leitlinien mit diesen Methoden untersucht werden sollten. Hier besteht dringender Handlungsbedarf für den Gemeinsamen Bundesausschuss, die Herz-CT und Herz-MRT in den Leistungskatalog der GKV aufzunehmen.

Professor Maintz, wenn Sie in einem Satz die wichtigste Aussage aus der genannten Umfrage nennen würden - welche wäre das?

Sobald die Herz-CT und Herz-MRT eine Regelleistung der Gesetzlichen Krankenversicherung werden, können wir diese Methoden flächendeckend auf hohem Qualitätsniveau anbieten - die Radiologie steht bereit! 